\title{
Effect of pectoralis minor relaxation on the prognosis of rotator cuff injury under arthroscopy
}

\author{
Shunling Chen, Di Yang, Quan Sun, Zhiyu Guan, Peiyong Tan, Kaiwei Zhang, Xiangfei Mao \\ Department of Orthopedics and Traumatology, the First Affiliated Hospital of Guizhou University of Traditional Chinese Medicine, Guiyang, China \\ Contributions: (I) Conception and design: S Chen, D Yang, K Zhang; (II) Administrative support: Q Sun; (III) Provision of study materials or patients: \\ Z Guan; (IV) Collection and assembly of data: Q Sun, Z Guan, P Tan, X Mao; (V) Data analysis and interpretation: S Chen, D Yang, K Zhang; (VI) \\ Manuscript writing: All authors; (VII) Final approval of manuscript: All authors. \\ Correspondence to: Di Yang. Department of Orthopedics and Traumatology, the First Affiliated Hospital of Guizhou University of Traditional Chinese \\ Medicine, Guiyang, China. Email: ydgzhtcm@126.com.
}

\begin{abstract}
Background Rotator cuff injury is the main cause of shoulder joint pain. Rotator cuff tear is a serious stage of rotator cuff injury. The purpose of this study was to observe the effect of pectoralis minor relaxation on the prognosis of rotator cuff injury under arthroscopy.

Methods: The clinical data of patients with rotator cuff injury admitted to our department from March 2019 to August 2020 were retrospectively analyzed. These patients were divided into a conventional arthroscopy surgery group and a pectoralis minor relaxation group. The American Shoulder and Elbow Surgeons' Form (ASES) and University of California at Los Angeles Shoulder Scores (UCLASS) were used to assess shoulder joint function during the postoperative and follow-up periods, and the visual analogue scale (VAS) was used to assess shoulder joint pain.
\end{abstract}

Results: Shoulder joint function of the two groups of patients was significantly improved at 1 week postoperatively compared with that before surgery $(\mathrm{P}<0.05)$. The shoulder joint function of the pectoralis minor relaxation group was better than that of the conventional arthroscopy surgery group $(\mathrm{P}<0.05)$. Also, the shoulder joint function of patients in pectoralis minor relaxation group was better than that in conventional arthroscopy surgery group at 6 weeks, 12 weeks and 6 months after surgery $(\mathrm{P}<0.05)$. The UCLASS evaluation results were consistent with the ASES results. At 1 week after surgery, the pain of the two groups of patients was significantly less than before the operation, but the degree of pain relief was greater at 6 weeks postoperatively. Starting at 6 weeks after surgery, the shoulder joint pain of the pectoralis minor relaxation group was less than that of conventional arthroscopy surgery group $(\mathrm{P}<0.05)$. The healing of the incision was observed after the operation. No incision infection or exudation was found in the two groups of patients.

Conclusions: The addition of pectoralis minor relaxation on the basis of conventional arthroscopic surgery is beneficial to the further recovery of shoulder joint function and pain reduction in patients with rotator cuff injury.

Keywords: Rotator cuff injury; pectoralis minor; arthroscopy; prognosis

Submitted Oct 31, 2021. Accepted for publication Jan 19, 2022.

doi: 10.21037/apm-21-3959

View this article at: https://dx.doi.org/10.21037/apm-21-3959

\section{Introduction}

Rotator cuff injury is the main cause of shoulder joint pain, especially in patients with degenerative shoulder joint lesion, and has also become a focus in sports medicine research in recent years. Rotator cuff tear is a serious stage of rotator cuff injury. At present, the main treatment is surgical repair of the torn tendon tissue to obtain anatomical integrity and restore the function of the rotator cuff. 
The movement of the scapula has long been considered a key component of shoulder movement, and the scapula is the main part connecting the trunk and the upper limbs. With our growing understanding of scapular kinematics and its role in shoulder pain and shoulder joint dysfunction, the pectoralis minor has attracted increasing attention. The pectoralis minor is crucial for patients with scapular dyskinesia, especially for patients with rotator cuff injury. At present, most patients with pectoralis minor tension achieve their rehabilitation goals through non-surgical treatment, stretching and strengthening of the pectoralis minor. However, the effect of non-surgical treatment in some patients is not ideal. Excessive tightness of the pectoralis minor will result in limited anterior scapular movement and downward inclination of the coracoid process, which is partly a cause of sick scapular syndrome (1). Simple pectoralis minor tightness can be treated non-surgically through static and dynamic stretching exercises (passive stretch maneuvers) $(2,3)$. In most patients, the symptoms of pectoralis minor tension can be relieved (3). However, for patients who cannot achieve satisfactory results, surgical relaxation of the pectoralis minor tendon may be a reasonable option, but related evidence is not currently sufficient. Only a few studies have reported that there are few adverse reactions related to surgical relaxation of the pectoralis minor, which has a certain effect and good safety (4).

Therefore, in this study, we explored the treatment effect and safety of pectoralis minor relaxation as an adjunct on the basis of conventional arthroscopic surgery for patients with rotator cuff tear and pectoralis minor tightness through retrospective analysis. We present the following article in accordance with the STROBE reporting checklist (available at https://apm.amegroups.com/article/view/10.21037/apm$21-3959 / \mathrm{rc})$.

\section{Methods}

\section{Study population}

In this study, we retrospectively enrolled patients with rotator cuff injury admitted to our department from March 2019 to August 2020. The inclusion criteria were as follows: (I) confirmed diagnosis of rotator cuff injury, combined with pectoralis minor tension; (II) patients aged $\geq 18$ years; (III) those who had received non-surgical treatment for more than 3 months, with poor results; (IV) patients with surgical indications including a rotator cuff tear width $>5 \mathrm{~mm}$, and a thickness that was more than half of the cuff; and (V) those who signed the surgical consent form and the clinical research informed consent form. The exclusion criteria were as follows: (I) patients with severe trauma that caused damage to the shoulder joint; (II) patients with malignant tumors that caused damage to the shoulder joint and surrounding tissues; (III) patients who had not received regular follow-up; and (IV) those with incomplete clinical data. According to the treatment methods, the patients were divided into a conventional arthroscopic surgery group (ASG) and a pectoralis minor relaxation group (PRG). All procedures performed in this study involving human participants were in accordance with the Declaration of Helsinki (as revised in 2013). The study was approved by the Ethics Committee of the First Affiliated Hospital of Guizhou University of Traditional Chinese Medicine (approval number: k2021-007) and informed consent was taken from all the patients.

\section{Assessment}

After admission, the patients underwent magnetic resonance imaging (MRI) examination, computed tomography (CT) examination, and other necessary examinations. The American Shoulder and Elbow Surgeons' Form (ASES) and University of California at Los Angeles Shoulder Scores (UCLASS) were used before and after surgery and during follow-up to score patients' shoulder joint function, and the visual analogue scale (VAS) was used to score patients' shoulder joint pain. According to the Neer staging of rotator cuff injury, the injuries were divided into the following: subacromial bursitis, rotator cuff tendinitis, and rotator cuff tear. The International Society of Arthroscopy, Knee Surgery and Orthopaedic Sports (ISAKOS) was used to distinguish the rotator cuff tear type in patients with rotator cuff tear, and the "Sick Scapula Rating Scale: Static Measurement" was used to quantitatively evaluate the sick scapula.

\section{Intervention}

In the process of receiving conventional decompression of the subacromial space under arthroscopy, the PRG received pectoralis minor relaxation under arthroscopy, while the ASG did not receive this procedure. The same rehabilitation program was conducted in all patients. We record the occurrence of complications, rechecked the blood cell count and hemoglobin the next day after surgery, observed the healing of the incision, and monitored the patients' blood pressure. 


\section{Arthroscopic operation of pectoralis minor relaxation}

Upon completion of the other surgical procedures, observation was carried out through the anterior lateral approach, and a low-temperature plasma knife was inserted into the anterior approach to release the deltoid muscle and reach the beak of the protruding medial. The arthroscopy was exchanged to the anterior approach to observe the inner surface of the coracoid process, and an auxiliary approach was established $1 \mathrm{~cm}$ inside the coracoid process under monitoring, and a low-temperature plasma knife was inserted to expose the pectoralis minor muscle. A lowtemperature plasma knife was used to paste the bone to loosen the pectoralis minor muscle, the pectoralis minor muscle was freed from the coracoid process, and the tissue near the stop point of the pectoralis minor muscle was taken for pathological examination to confirm the internal pathological changes of the pectoralis minor muscle. During the operation, attention was paid not to damage the brachial plexus and axillary artery on the deep surface of the pectoralis minor muscle.

\section{Follow-up}

The patients were followed up at 1, 6, and 12 weeks, 6 months and 1 year postoperatively, and the shoulder function (ASES, UCLASS) and pain (VAS) scores were recorded. MRI was conducted to investigate the healing of the rotator cuff injury.

\section{Statistical analysis}

Statistical analysis was performed using SPSS statistical software (version 22.0, IBM, Chicago, USA). Quantitative data in accordance with the normal distribution were represented by mean \pm standard deviation, and comparison between groups was conducted using the $t$-test. Qualitative data were represented by quantity (percentage), and comparison between groups was conducted using the $\chi^{2}$ test. $\mathrm{P}<0.05$ was considered to indicate a statistically significant difference.

\section{Results}

\section{Baseline characteristics}

According to the inclusion and exclusion criteria, this study included 30 patients with rotator cuff injury, with 15 cases each in the PRG and ASG (Table 1). Patients in this study were $25-69$ years old, with an average of $50.5 \pm 11.5$ years. There were seven males (23.3\%) and 23 females (76.7\%). According to the Kibler classification, 16 cases (53.3\%) were type I, seven cases were type II (23.3\%), five cases were type III (16.7\%), and two cases were type IV (6.7\%). There was no statistical difference in the baseline characteristics of the two groups of patients $(\mathrm{P}>0.05)$.

\section{Comparison of postoperative changes in shoulder joint function}

We used the ASES and UCLASS scores to evaluate shoulder joint function (Table 2). The ASES evaluation results showed that there was no statistical difference in shoulder joint function between the two groups of patients before surgery. At 1-week postoperatively, the shoulder joint function of the two groups was significantly improved compared with that before surgery $(\mathrm{P}<0.05)$. Moreover, the shoulder joint function of patients in the PRG was superior to that of the ASG $(\mathrm{P}<0.05)$, as shown in Figure 1.

We also compared the two groups of patients 6 weeks after surgery with 1 week after surgery, 12 weeks after surgery with 6 weeks after surgery, and 6 months after surgery with 12 weeks after surgery. The results showed that the shoulder joint function of the two groups of patients has further improved, and during the same period, the shoulder joint function of patients in the PRG was better than that of the ASG $(\mathrm{P}<0.05)$. However, the shoulder joint function of the two groups of patients at 12 months after surgery did not improve further than that at 6 months $(P>0.05)$, while the shoulder joint function of patients in the PRG was better than that of the ASG $(\mathrm{P}<0.05)$. The UCLASS evaluation results were basically consistent with the ASES results.

\section{Comparison of postoperative shoulder pain before and after surgery}

We used VAS to evaluate the patients' shoulder joint pain. There was no significant difference in the local pain of the shoulder joint between the two groups of patients before the operation $(\mathrm{P}>0.05)$. The pain of patients in this study was significantly less than before the operation; it was alleviated to a greater extent at 6 weeks postoperatively, and was still further relieved at the 6 months after surgery. No further improvement was observed at 12 months after the operation (Table 3). During the same period of evaluation, there was no significant difference in pain between the two groups at 
Table 1 Comparison of baseline data $(\mathrm{n}=30)$

\begin{tabular}{|c|c|c|c|c|}
\hline Characteristics & PRG $(n=15)$ & ASG $(n=15)$ & $t / \chi^{2}$ value & $P$ value \\
\hline Female (n, \%) & $11(73.3)$ & $12(80.0)$ & 0.186 & 0.666 \\
\hline BMI $\left(\mathrm{kg} / \mathrm{m}^{2}\right)$ & $23.6 \pm 2.0$ & $24.4 \pm 3.2$ & 0.884 & 0.384 \\
\hline Kibler classification (n, \%) & & & 0.593 & 0.898 \\
\hline II & $3(20.0)$ & $4(26.7)$ & & \\
\hline III & $2(13.3)$ & $3(20.0)$ & & \\
\hline IV & $1(6.7)$ & $1(6.7)$ & & \\
\hline WBC $\left(\times 10^{9} / L\right)$ & $5.6 \pm 0.8$ & $5.7 \pm 0.6$ & 0.466 & 0.645 \\
\hline PLT (×10/L) & $145.3 \pm 9.1$ & $155.1 \pm 10.0$ & 0.165 & 0.870 \\
\hline ALT (U/L) & $30.4 \pm 8.3$ & $30.7 \pm 7.0$ & 0.104 & 0.918 \\
\hline $\mathrm{Cr}(\mu \mathrm{mol} / \mathrm{L})$ & $52.0 \pm 13.3$ & $52.5 \pm 13.2$ & 0.101 & 0.921 \\
\hline SBP $(m m H g)$ & $131.5 \pm 14.3$ & $130.3 \pm 14.5$ & 0.215 & 0.831 \\
\hline HR (beats per minute) & $70.9 \pm 3.7$ & $70.4 \pm 4.7$ & 0.303 & 0.764 \\
\hline
\end{tabular}

PRG, pectoralis minor relaxation group; ASG, arthroscopic surgery group; BMI, body mass index; WBC, white blood cell; NEU, neutrophil; $\mathrm{Hb}$, hemoglobin; PLT, platelet; ALT, alanine aminotransferase; Cr, creatine; SBP, systolic blood pressure; HR, heart rate.

Table 2 Comparison of postoperative shoulder joint function

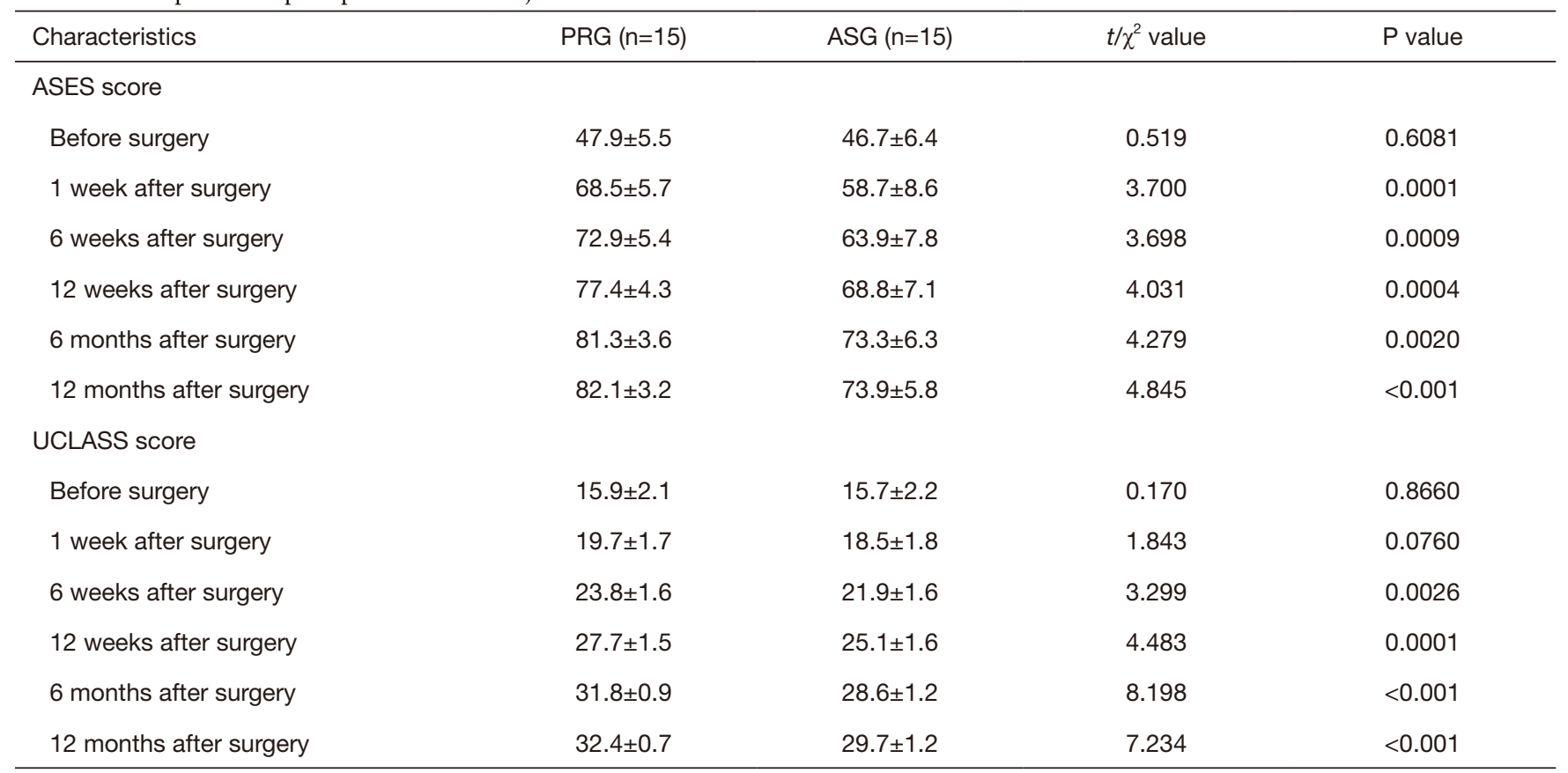

PRG, pectoralis minor relaxation group; ASG, arthroscopic surgery group; ASES, American Shoulder and Elbow Surgeons; UCLASS, University of California at Los Angeles Shoulder Scores. 

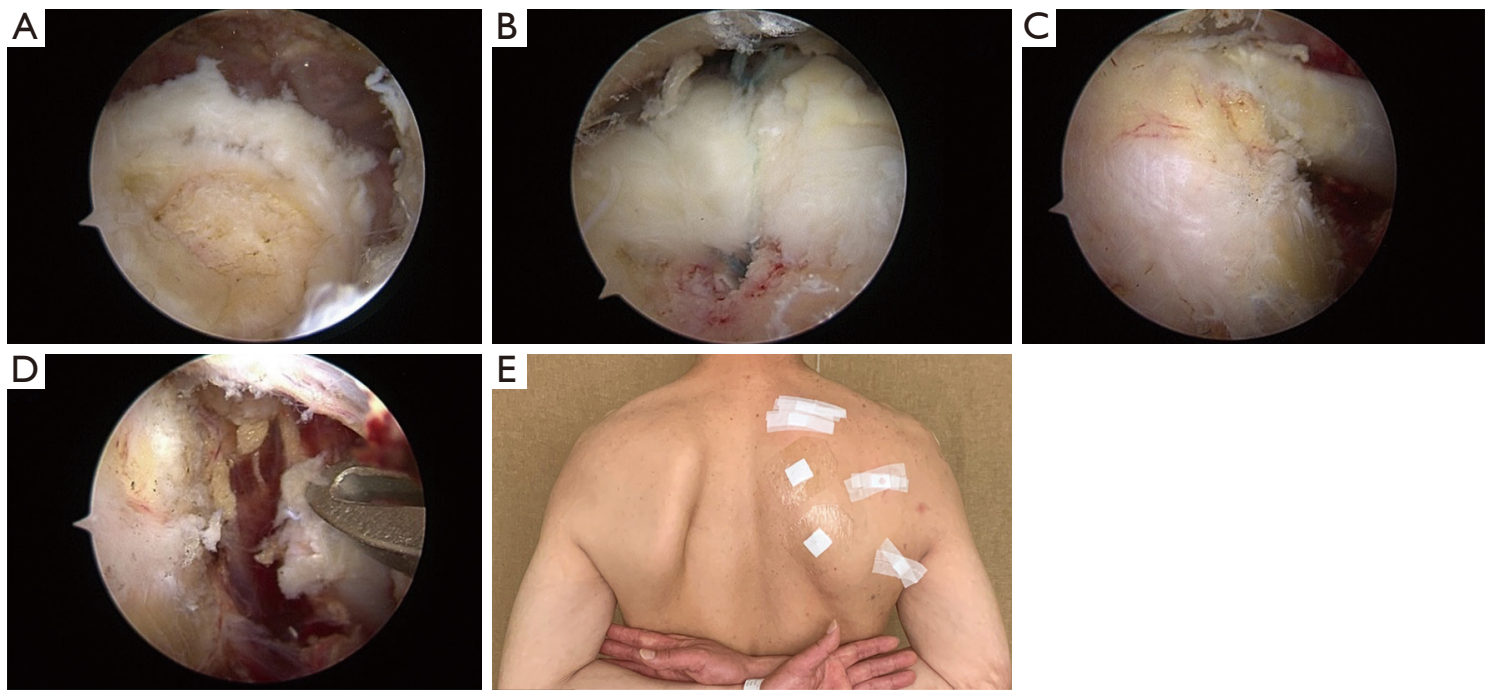

Figure 1 Arthroscopy findings during operation (A-D) and change of postoperative subscapular angle (E). (A) Arthroscopy shows a tear of the supraspinatus muscle. (B) Repair of the supraspinatus muscle under arthroscopy. (C) Exposure of the pectoralis minor tendon under arthroscopy. (D) Loosening of the pectoralis minor tendon under arthroscopy. (E) The postoperative subscapular angle uplift was significantly improved compared with that preoperatively.

Table 3 Comparison of postoperative shoulder joint pain (VAS score)

\begin{tabular}{|c|c|c|c|c|}
\hline Characteristics & PRG $(n=15)$ & ASG $(n=15)$ & $t / \chi^{2}$ value & $P$ value \\
\hline 1 week after surgery & $6.3 \pm 0.7$ & $6.2 \pm 0.8$ & 0.487 & 0.630 \\
\hline 6 weeks after surgery & $3.0 \pm 0.9$ & $3.6 \pm 0.5$ & 2.201 & 0.036 \\
\hline 12 weeks after surgery & $1.3 \pm 0.5$ & $1.8 \pm 0.7$ & 2.168 & 0.039 \\
\hline 12 months after surgery & $0.3 \pm 0.5$ & $0.7 \pm 0.5$ & 2.316 & 0.028 \\
\hline
\end{tabular}

VAS, visual analogue scale; PRG, pectoralis minor relaxation group; ASG, arthroscopic surgery group.

1 week after the operation; however, from 6 weeks after the operation, the PRG had less shoulder pain compared to the ASG $(\mathrm{P}<0.05)$.

\section{Safety comparison}

We monitored the patients' blood pressure for three consecutive days after surgery. The results showed that there was no significant change in systolic blood pressure between the two groups compared to the preoperative values $(\mathrm{P}>0.05)$, and there was no statistical difference between the two groups $(\mathrm{P}>0.05)$. The blood cell count showed that the white blood cell, neutrophil, and red blood cell counts of the two groups of patients had no significant changes compared with before the operation. Also, the hemoglobin content was not significantly reduced, and there was no statistical difference between the two groups $(\mathrm{P}>0.05)$. Moreover, incision healing was observed postoperatively, and no incision infection or exudation was found in the two groups of patients.

\section{Discussion}

This study retrospectively analyzed the clinical data of patients who underwent rotator cuff injury surgery and postoperative follow-up. We found that compared with 
conventional arthroscopic surgery, pectoralis minor muscle relaxation can further improve joint function which was the biggest advantage. Patients with diseased shoulder joint function can relieve local shoulder joint pain to a greater extent without increasing the risk of intraoperative/ postoperative bleeding as well as infection in the shoulder joint cavity and incision site. Further analysis showed that the addition of pectoralis minor relaxation could further enhance the improvement of the patient's shoulder joint function after surgery, until it stabilizes at 6 months after surgery, and is better than conventional arthroscopic surgery alone. It also has a similar sustained effect on pain relief. The problem should be taken into consideration is that this intervention is also a minor injury.

Rotator cuff injuries are very common, especially in the elderly (1,5-7). The development of arthroscopic technology has made the surgical treatment of rotator cuff injuries more acceptable to patients, greatly improving the shoulder joint function and quality of life of patients (8-10). Studies have found that the pectoralis minor also plays a role in joint dysfunction after rotator cuff injury. The pectoralis minor originates from the anterior surface of the third to fifth ribs and inserts into the inner side of the upper surface of the coracoid process of the scapula, and its main function is to stabilize the scapula and adjust forward tilt (11). The pectoralis minor pulls the coracoid process forward toward the chest wall, so it is an important internal rotator of the scapula.

The optimization of motor functions is essential for correct shoulder kinematics and human posture. According to reports, changes in length, function, and elasticity can cause abnormalities in the mechanics of the scapula and even the shoulder joint, pain, and shoulder joint dysfunction. The tightness of the pectoralis minor can interfere with the normal movement of the scapula and may cause shoulder pain, accompanied by tenderness inside the coracoid process (12). According to reports, the pathological tension of the pectoralis minor muscle can cause the height of the subacromial space to narrow during overhead movement, resulting in impact between the coraco-acromial arch and the rotator cuff tendons, which in turn leads to an increased risk of rotator cuff injury (11). The cause of this disease may be excessive force of the chest muscles during heavy lifting or repeated overhead activities. Burkhart et al. introduced the concepts of abnormal scapular position, inner and lower scapular border bulge, coracoid pain and dislocation, and scapular dyskinesia syndrome, known as SICK scapular (Scapular malposition,
Inferior medial border prominence, Coracoid pain and malposition, and dysKinesis of scapular movement) (13). Sick scapular is a serious stage of scapular motility disorder, which is divided into three types (with type II being mostly related to labrum injury): type $\mathrm{I}$, the inner and lower edge of the scapula is prominent; type II, the medial edge of the scapula is prominent; and type III manifests as a bulge on the medial edge of the scapula, which is related to the height of the rotator cuff injury. Their study showed that Sick scapular syndrome is the cause of pain in the front of the shoulder, upper back of the shoulder blade, upper shoulder, as well as the proximal and outer upper arm (13). There are few reports that are similar to our study. One report showed that the treatment of patients with Sick scapular syndrome who failed non-surgical treatment through pectoralis minor muscle relaxations achieved satisfactory results (14). Another study reported that the treatment of patients with Sick scapular syndrome who failed non-surgical treatment by releasing the pectoralis minor under arthroscopy also achieved satisfactory results (15). Neither of these reports showed obvious complications. Unfortunately, there is no report on the study of Sick scapular syndrome in patients with rotator cuff injury. In our clinical practice, we found that many patients with rotator cuff injuries, especially patients with rotator cuff tears, are repaired by surgical treatment for the incompleteness of the rotator cuff, and at the same time, surgical methods are used to remove the subacromial bone, subacromial scars, thickened inflammatory bursae, following by rehabilitation training. There are still some patients with poor shoulder pain relief prognosis, slow recovery progress, and even nonhealing or tearing of the rotator cuff tendon during the postoperative follow-up. Among them, most of these are patients with diseased scapula, especially type III diseased scapula. Some patients do not have the conditions to receive effective scapular posture assessment, some patients live far away from the hospital or cannot receive systematic scapular dynamic training due to economic reasons, and some patients cannot correct the Sick scapula through nonsurgical treatment. Our research showed that there is a close relationship between Sick scapular and rotator cuff injury. Pectoralis minor relaxation can effectively treat Sick scapular, thereby improving the treatment effect of rotator cuff injury. Despite the positive result of our study, we recommend patients with rotator cuff injury to avoid heavy lifting and external impact on the shoulder.

There were some limitations in this study that should be noted. Firstly, this study is a preliminary study with a 
small sample size. Also, this study failed to further analyze whether there are differences in the therapeutic effects of pectoralis minor muscle relaxation on different types of rotator cuff injuries. At the same time, this study is a retrospective study, and the examination data of some patients was missing, so a more in-depth analysis could not be carried out. The next step of the study could be a multicenter, prospective, randomized controlled study, and longterm follow-up to further observe the mid- and long-term efficacy and safety of pectoralis minor muscle relaxation for patients with rotator cuff injury, as well as its impact on the quality of life of patients.

\section{Acknowledgments}

Funding: None.

\section{Footnote}

Reporting Checklist: The authors have completed the STROBE reporting checklist. Available at https://apm. amegroups.com/article/view/10.21037/apm-21-3959/rc

Data Sharing Statement: Available at https://apm.amegroups. com/article/view/10.21037/apm-21-3959/dss

Conflicts of Interest: All authors have completed the ICMJE uniform disclosure form (available at https://apm. amegroups.com/article/view/10.21037/apm-21-3959/coif). The authors have no conflicts of interest to declare.

Ethical Statement: The authors are accountable for all aspects of the work in ensuring that questions related to the accuracy or integrity of any part of the work are appropriately investigated and resolved. All procedures performed in this study involving human participants were in accordance with the Declaration of Helsinki (as revised in 2013). The study was approved by the Ethics Committee of the First Affiliated Hospital of Guizhou University of Traditional Chinese Medicine (approval number: k2021007) and informed consent was taken from all the patients.

Open Access Statement: This is an Open Access article distributed in accordance with the Creative Commons Attribution-NonCommercial-NoDerivs 4.0 International License (CC BY-NC-ND 4.0), which permits the noncommercial replication and distribution of the article with the strict proviso that no changes or edits are made and the original work is properly cited (including links to both the formal publication through the relevant DOI and the license). See: https://creativecommons.org/licenses/by-nc-nd/4.0/.

\section{References}

1. Watanabe A, Ono Q, Nishigami T, et al. Differences in Risk Factors for Rotator Cuff Tears between Elderly Patients and Young Patients. Acta Med Okayama 2018;72:67-72.

2. Borstad JD, Ludewig PM. Comparison of three stretches for the pectoralis minor muscle. J Shoulder Elbow Surg 2006;15:324-30.

3. Williams JG, Laudner KG, McLoda T. The acute effects of two passive stretch maneuvers on pectoralis minor length and scapular kinematics among collegiate swimmers. Int J Sports Phys Ther 2013;8:25-33.

4. Mercier N, Saragaglia D. Mini-open latarjet procedure for recurrent anterior shoulder instability. Adv Orthop 2011;2011:656205.

5. Smith MA, Smith WT. Rotator cuff tears: an overview. Orthop Nurs 2010;29:319-22; quiz 23-4.

6. Fang Y, Zhang Q. Outcomes after rotator cuff repair in the elderly as assessed by the American Shoulder and Elbow Surgeons shoulder score. Clinics (Sao Paulo) 2020;75:e1817.

7. Rogers BA, Little NJ, Ricketts DM. The management of rotator cuff tears in the elderly. J Perioper Pract 2012;22:30-4.

8. Thigpen CA, Shaffer MA, Gaunt BW, et al. The American Society of Shoulder and Elbow Therapists' consensus statement on rehabilitation following arthroscopic rotator cuff repair. J Shoulder Elbow Surg 2016;25:521-35.

9. Gilotra M, O'Brien MJ, Savoie FH, 3rd. Arthroscopic Rotator Cuff Repair: Indication and Technique. Instr Course Lect 2016;65:83-92.

10. Sheean AJ, Hartzler RU, Burkhart SS. Arthroscopic Rotator Cuff Repair in 2019: Linked, Double Row Repair for Achieving Higher Healing Rates and Optimal Clinical Outcomes. Arthroscopy 2019;35:2749-55.

11. Lewis JS, Valentine RE. The pectoralis minor length test: a study of the intra-rater reliability and diagnostic accuracy in subjects with and without shoulder symptoms. BMC Musculoskelet Disord 2007;8:64.

12. Kibler WB, McMullen J. Scapular dyskinesis and its relation to shoulder pain. J Am Acad Orthop Surg 2003;11:142-51.

13. Burkhart SS, Morgan CD, Kibler WB. The disabled 
throwing shoulder: spectrum of pathology Part III: The SICK scapula, scapular dyskinesis, the kinetic chain, and rehabilitation. Arthroscopy 2003;19:641-61.

14. Provencher MT, Kirby H, McDonald LS, et al. Surgical Release of the Pectoralis Minor Tendon for Scapular Dyskinesia and Shoulder Pain. Am J Sports Med

Cite this article as: Chen S, Yang D, Sun Q, Guan Z, Tan P, Zhang K, Mao X. Effect of pectoralis minor relaxation on the prognosis of rotator cuff injury under arthroscopy. Ann Palliat Med 2022;11(1):77-84. doi: 10.21037/apm-21-3959
2017;45:173-8.

15. Hendrix ST, Hoyle M, Tokish JM. Arthroscopic Pectoralis Minor Release. Arthrosc Tech 2018;7:e589-94.

(English Language Editor: A. Kassem) 